\title{
Antibiotic prophylaxis for transrectal ultrasound-guided prostate needle biopsy: Compared efficacy of ciprofloxacin vs. the ciprofloxacin/fosfomycin tromethamine combination
}

\author{
Alexandre Morin ${ }^{1}$; Marco Bergevin ${ }^{2}$; Natalie Rivest ${ }^{2}$; Steven P. Lapointe ${ }^{3}$ \\ ${ }^{1}$ Faculté de médecine et des sciences de la santé, Université de Sherbrooke, QC, Canada; ${ }^{2}$ Department of \\ Microbiology, Cité-de-la-Santé Hospital, Laval, affiliated with Université de Montréal, Montreal, QC, Canada; \\ ${ }^{3}$ Urology Service UroLaval, Cité- de-la-Santé Hospital, Laval and St-Eustache Hospital, affiliated with Université de \\ Montréal, Montreal, QC, Canada
}

Acknowledgements/funding: The authors would like to thank the urologists at UroLaval, Drs. Mathieu Bettez, Jean Cossette, Benoit Guertin, Samer Hanna, Bechir Hage, Marie-Paule Jammal, and Jean Simard. They also want to specially thank Dr. Alex Carignan for reviewing this article and the Sherbrooke University-affiliated hospital research centre statistician team for the statistical calculations. This research was made possible by an unrestricted educational grant from Sanofi.

Cite as: Can Urol Assoc J 2020 March 23; Epub ahead of print. http://dx.doi.org/10.5489/cuaj.6248

Published online March 23, 2020

$* * *$

\section{Abstract}

Introduction: Some authors advocate an increase in post-prostate needle biopsy (PNB) infections associated with emergent quinolone resistance in E. coli, urging re-evaluation of antibioprophylaxis. In this study, we compared rates of post-PNB urosepsis associated with two oral regimens of antibioprophylaxis: ciprofloxacin (CIP) vs. ciprofloxacin and fosfomycin tromethamine combination (CIP/FOS).

Methods: This retrospective pre-post intervention study included all patients who underwent PNB in two Canadian hospitals from January 2012 to December 2015. The primary outcome was urosepsis within one month of PNB. Urosepsis rates were analyzed according to antibioprophylaxis using log-binomial regression, considering the propensity scores weights of collected risk factor data.

Results: We reviewed 2287 PNB patients. A total of 1090 received CIP and 1197 received CIP/FOS. Urosepsis incidence with CIP was $1.1 \%$ (12/1090) and fell to $0.2 \%$ (2/1197) with CIP/FOS. Our analysis indicates that CIP/FOS significantly decreased the risk of urosepsis compared to CIP alone (adjusted relative risk [aRR] 0.16; $\mathrm{p}=0.021$ ). The isolated pathogen was E. coli in 12/14 cases, including seven bacteremias. Among E. coli cases, seven strains were CIP- 
resistant. Eleven of 12 E. coli, including all CIP-resistant strains, were isolated in patients on CIP alone. One case of $B$. fragilis bacteremia occurred in the CIP/FOS group. No cases of $C$. difficile infection were identified in the three months post-PNB.

Conclusions: The adoption of CIP/FOS antibiotic prophylaxis significantly lowered the rate of post-PNB urosepsis. Conveniently, this regimen is oral, single-dose, and low-cost.

\section{Introduction}

Transrectal ultrasound-guided prostate needle biopsy (PNB) is a useful procedure for prostate cancer diagnosis and pathological staging ${ }^{1}$. Its main complications are macrohematuria, rectal bleeding, urinary retention, erectile dysfunction, needle tract seeding, and hematospermia. Infectious complications include cystitis, epididymitis, orchitis, prostatitis and urosepsis ${ }^{1,2}$. Although PNB is generally considered a safe procedure, infectious complication rates range from $0.1 \%$ to $7 \%$ and up to $3.1 \%$ of patients were noted to develop urosepsis ${ }^{2,3}$. The American Urology Association guidelines therefore recommend the use of antibiotic prophylaxis, more specifically fluoroquinolones (FQs) or $1^{\text {st }}, 2^{\text {nd }}$ or $3^{\text {rd }}$ generation cephalosporins as first line antibioprophylaxis, at least $1 \mathrm{~h}$ prior to the procedure ${ }^{2}$.

Recent studies report rising rates of post-PNB infections associated with emergent FQ resistant E. coli and extended-spectrum $\beta$-lactamase producing E. coli (ESBL), raising concerns about efficacy of these antibioprophylaxis regimens ${ }^{2,4-6}$. On the other hand, various mitigating strategies are suggested in the literature: rectal swab screening with directed antibioprophylaxis $^{7,8}$, switching antibioprophylaxis ${ }^{7,9-11}$ and transperineal biopsy approach ${ }^{2,7}$. Recently, some authors pointed out fosfomycin tromethamine (FOS) as being an attractive alternative, because of its adequate levels in prostatic tissue obtained with a single $3 \mathrm{~g}$ oral dose, the low level of bacterial resistance, its broad spectrum of activity including multidrug resistant organisms, and its good safety profile ${ }^{10-12}$.

A series of post-PNB infections and the above mentioned FOS characteristics prompted us to modify our antibioprophylaxis regimen. The objective of our study was to compare rates of post-PNB urosepsis associated with 2 oral regimens of antibioprophylaxis: ciprofloxacin (CIP) vs. ciprofloxacin and fosfomycin tromethamine combination (CIP/FOS).

\section{Methods}

\section{Study design and population}

We conducted a retrospective pre-post intervention study in two university affiliated Canadian hospitals. In December 2013, CIP antibioprophylaxis was augmented to CIP/FOS combination. This antibioprophylaxis has, since then, become the new standard of care in our centers. 
Approval from our institutional ethical review board was obtained for this study (2016-2017/0401-A C112).

The population was composed of all patients who underwent a PNB from January 2012 to December 2015 in these two centers and an affiliated outpatient clinic. Patients who did not receive either CIP or CIP/FOS combination for antibioprophylaxis prior to the biopsy were excluded. Group 1 was composed of patients who received oral ciprofloxacin $500 \mathrm{mg} 2$ hours prior to the PNB, along with a sodium phosphate enema (January 2012 to November 2013). Group 2 was composed of patients who received oral ciprofloxacin $500 \mathrm{mg}$ and oral fosfomycin tromethamine $3 \mathrm{~g} 2$ hours prior to the PNB, along with a sodium phosphate enema (December 2013 to December 2015).

\section{Biopsy procedure}

PNBs were performed either in an outpatient urology clinic or in the endoscopy suite at the hospital by one of the 9 certified Canadian urologists in our team. Patients were instructed to selfadminister antibioprophylaxis and sodium phosphate enema 2 hours prior the procedure. Before the biopsy, urologists systematically verified if patients had taken their antibioprophylaxis and if they had specific infection symptoms (i.e. fever, chills or lower urinary tract symptoms, such as urgency, frequency, dysuria or suprapubic tenderness). If patients were symptomatic or did not follow protocol, PNB was postponed.

Before the procedure, trans-rectal periprostatic local anaesthetic was performed using lidocaine. With patients on the left-lying position, we performed trans-rectal ultrasound guided prostate biopsy with a 10 to 12 -core strategy, with the use of a Pro-mag Ultra automatic biopsy instrument with a disposable 18 -gauge $\times 20-25 \mathrm{~cm}$ biopsy Argon pro-mag needle. All patients were instructed to seek medical attention at our two hospitals if they developed symptoms related to biopsy complication (i.e. severe bleeding, urinary retention, fever, chills or lower urinary tract symptoms).

\section{Data collection}

Electronic medical records of all patients were reviewed for demographic, clinical and microbiological data. Clinical information included emergency consultations, hospital admissions and risk factors for post-PNB infection distributed into comorbidities, infectious and urological risk factors $3,4,7,10,13$. Microbiological data included available midstream-voided urine and blood cultures as well as the pathogens and antibiotic susceptibility testing, as reported by clinical microbiological laboratories. Urine or blood cultures were only performed for symptomatic patients who presented at the emergency ward. We also evaluated development of C. Difficile colitis up to 3 months post-PNB.

The main outcome was urosepsis defined by urinary tract infection (UTI) with bacteremia or as UTI with systemic inflammatory response syndrome (SIRS) ${ }^{14}$ within 1 month of PNB. The 
clinical impacts of infectious complications were also documented, and included 30-day mortality, vasopressor requirement, length of hospitalization, and length of stay at the intensive care unit.

Non-infectious complications were similarly documented and were graded according to the severity. Grade 1 included macrohematuria, hematospermia and dysuria and grade 2 included acute urinary retention, significant rectal bleeding and prostatic hematoma ${ }^{10}$. 


\section{Statistical analysis}

To assess the risk factors' statistical significance either T test, Mann-Whitney test, Fisher's exact test or Chi-squared test were used as appropriate. For urosepsis incidence in the CIP and CIP/FOS groups, Wilson's 95\% confidence intervals were used.

To account for baseline differences between the cohorts, inverse probability of treatment weighting adjusted analyses were performed. The propensity score was estimated using a logistic regression model. The comparison of infection rates between the two cohorts was made using log-binomial regression considering the propensity score weights, with $p<0.05$ marking statistical significance.

\section{Results}

A total of 2304 patients were assessed for eligibility. We excluded 17 patients from the study for incomplete demographic data: 11 in the CIP group, 6 in the CIP/FOS group. CIP group was left with 1090 patients and CIP/FOS group with 1197 patients. No infectious complications were found amongst the excluded patients upon chart review.

All patient clinical and laboratory characteristics at the time of the biopsy are found in table 1 . There were significant differences between the two study periods. More patients underwent cystoscopies in the month prior to PNB and repeat biopsies in the first group, while higher level prostatic-specific antigen levels and more aggressive prostate cancer histological findings were observed in the second group. There was also a difference in the local antibiogram for CIP susceptible E. coli rates between the two cohorts: it dropped from $86.8 \%$ to $85.1 \%$. In the month following PNB, 14 urosepsis cases were identified in the entire study population. The median hospital stay was 2 days (interquartile range [IQR]: $1-3.25 \mathrm{~d}$ ). One patient was sent to the ICU, none required the use of vasopressors and there was no mortality within 30 days of PNB. A total of 13 patients received intravenous antibiotics during their hospitalisation. The median time between biopsy and emergency consultation was 1 day (range: $0 \mathrm{~d}-24 \mathrm{~d}$ ).

Overall incidence was 1.1 urosepsis per 100 biopsies (95\% CI: $0.63 \%-1.91 \%$ ) in the CIP group (12/1090 patients). Of these cases, 7 had bacteremia. E. coli was the only pathogen. All the FQ-resistant and ESBL E. coli strains were found in this group. In the CIP/FOS group, 2 urosepsis events were identified (2/1197) patients leading to an incidence of $0.2 \%(95 \% \mathrm{CI}$ : $0.05 \%-0.61 \%$ ). There was one case of E. coli urosepsis and one case with Bacteroides fragilis bacteremia. Detailed microbiological characteristics of cases are found in table 2.

In the multivariate analysis, the urosepsis incidence rate was significantly lower in the CIP/FOS combination antibioprophylaxis group, giving an adjusted relative risk (aRR) of 0.16 (95\% CI: $0.03-0.76 ; P=0.02)$. 


\section{Other complications}

1 case of prostatitis, not meeting urosepsis criteria, with no identified pathogen was also found in the CIP group. This patient was excluded from analysis.

In patients who consulted at the hospital after PNB, there were a total of 4/1090 (0.4\%) grade 1 and 10/1090 (0.9\%) grade 2 post-PNB non-infectious complications amongst the CIP group. As for the CIP/FOS group, there were $1 / 1197(0.1 \%)$ grade 1 and 3/1197 (0.3\%) grade 2 noninfectious complications. All reported post-procedural non-infectious complications are displayed in table 3.

No cases of $C$. difficile infection were identified in either group in the 3 months post administration of antibioprophylaxis.

\section{Discussion}

Our study demonstrates that the CIP/FOS combination was associated with an $84 \%$ decrease in the incidence of urosepsis when compared to a CIP regimen. This reduction was statistically significant despite a low baseline incidence rate of post-PNB urosepsis and an increase in E. coli resistance in our centers throughout the study period.

In recent years, FQ antibioprophylaxis regimen used to cover most relevant urological pathogens ${ }^{15}$. However, many studies report increased post-PNB infections associated with increasing rates of FQ resistant $E$. coli $^{2,4-6}$. Considering there is an estimate of $>1$ million PNB annually in the United States ${ }^{16}$ and that urosepsis can lead to hospitalization and serious health hazards ${ }^{2}$ changes in antibioprophylaxis regimen need to be re-assessed.

We observed such an increase in CIP resistant E. coli at our institutions as well as an increase in post-PNB infections, especially in 2013. This prompted us to change our antibioprophylaxis regimen to the combination of CIP/FOS. At the time, there were no published studies on the use of FOS alone. We therefore elected to use CIP for its excellent enterobacteriæ coverage ${ }^{15}$ and to add FOS to cover the CIP resistant strains.

There were no cases of CIP resistant or ESBL E. coli urosepsis in the CIP/FOS group, suggesting that FOS covered these multidrug resistant pathogens. The wider spectrum of antimicrobial coverage afforded by this regimen could have led to increased post-PNB C. difficile cases, but none were identified in the 3 months post-procedure. The negligible influence of FOS on the microbiome when compared to other broad-spectrum antimicrobial agents and the lower propensity for significant microbiome disruption with single dose regimens could explain this finding ${ }^{11}$.

Theoretically, the use of FOS alone, considering its pharmacokinetic characteristics and its broad spectrum, is a rational option for standalone antibioprophylaxis ${ }^{11}$. Even more so, many reports indicate low reported resistance rates to relevant pathogens ${ }^{12,17}$. According to a CANWARD surveillance prospective study on FOS susceptibility rates conducted from 2010 to 2013, 99.4\% of 868 urinary E. coli isolates were susceptible to FOS $^{18}$. 
We have identified two European studies ${ }^{10,19}$ published after our regimen choice that suggest standalone FOS as a viable option for antibioprophylaxis. There was also a meta-analysis by Roberts et al. $(n=3112)$ that has demonstrated a significant reduction in post-PNB grade 2 infections (bacteremia, febrile UTI, urosepsis) with FOS, giving an adjusted odds ratio (aOR) of $0.13(95 \% \mathrm{CI}: 0.07-0.26)^{11}$. Their results reassuringly correlate with our own and even question the relevance of maintaining CIP in the CIP/FOS combination.

However, in one large Canadian study $(n=9527)$, use of single dose FOS for antibioprophylaxis resulted in an increased risk of infectious complications compared to CIP alone (aOR: 1.80; 95\% CI: $1.10-2.94 ; P=0.02)$. This increased risk was not corrected by adding a second dose of FOS $12 \mathrm{~h}$ post-PNB (aOR: 1.43 with $95 \% \mathrm{CI}$ : $0.66-3.09$ and $P$ value: 0.36$)^{20}$. A Klebsiella spp. breakthrough, most likely justified by the poor FOS coverage for this pathogen ${ }^{12}$, could explain this increased risk.

Before choosing the CIP/FOS combination as antibioprophylaxis for PNB in our centers, other mitigating strategies have also been contemplated. As advocated by some authors, we considered using rectal swab screening to direct antibioprophylaxis prior to $\mathrm{PNB}^{7,8}$. However, this strategy has mixed results in the literature. Some authors point out no difference in post-PNB infectious complications with targeted antibioprophylaxis ${ }^{21-25}$, along with an increased burden for clinical microbiology laboratories and urologists ${ }^{4}$. Also, it has been demonstrated that in a single

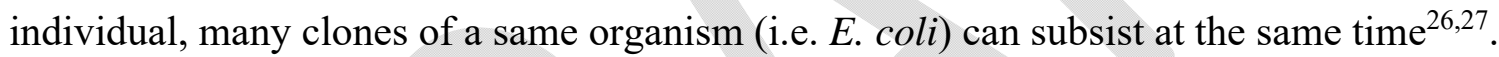
Antibioprophylaxis is therefore, most often, targeted only towards CIP resistant clones and could neglect the resistance patterns of other clones.

Another approach would be to use intravenous or intramusclar antibiotics, such as gentamicin or amikacin ${ }^{2,4,9,12}$. According to one large study conducted by Jiang et al. $(\mathrm{n}=15236)$ augmented antibiotic prophylaxis composed of CIP and intramuscular gentamicin or intramuscular amikacin significantly reduced the rate of post-PNB urosepsis when compared to standalone CIP or targeted antibiotic prophylaxis (OR 0.35, 95\% CI $0.16-0.76, \mathrm{p}=0.008)^{21}$. Many antibiotic prophylaxis regimens, such as this one, have demonstrated that they can diminish post-PNB infections, and can add protection for CIP resistant $E$. coli ${ }^{2,9,10}$. Unfortunately, these are administered either intra-muscularly or intravenously, which is associated with an increased cost and logistical burden.

A transperineal approach for PNB has also been shown to diminish infectious complications and rectal bleeding, but it has a similar post-procedural hospitalization rate due to increased acute urinary retention ${ }^{3,7,28}$. Moreover, widespread adoption of transperineal approach was thought to be nearly impossible and too expensive, as it requires hospitalization and general anaesthesia for all patients. However, some authors have recently advocated in-office transperineal prostate biopsy under local anesthesia without antibioprophylaxis. This approach was demonstrated to be safe, effective and well tolerated by patients, despite some concern over 
post-procedural urinary retention ${ }^{29,30}$. This alternative should be strongly considered, as it would nearly eliminate the need for antibioprophylaxis, therefore supporting antimicrobial stewardship. Until in-office, antibioprophylaxis free transperineal prostate biopsies are widely accepted, it is our view that antibiotic prophylaxis regimens with low baseline resistance in target pathogens are preferable. Thus, FOS represents an excellent antibiotic alternative, which can be administered orally. Therefore, the use of CIP/FOS combination antibioprophylaxis represents a valid and practical alternative to the above-mentioned strategies.

\section{Strengths and limitations}

Our large study group enabled us to demonstrate statistically significant differences in urosepsis between the two regimens, despite its low incidence rate. The measured effect of an adjusted risk reduction of 0.16 is clinically significant but the small number of urosepsis cases leaves us with a relatively wide confidence interval $(95 \% \mathrm{CI}: 0.03-0.76)$. The true effect may not be as notable as the measured effect, but is at worst end of our confidence interval compatible with a $24.4 \%$ decrease in post-PNB urosepsis rates, which we consider clinically meaningful.

This study also has several limitations. First, this non-randomized and retrospective study has an inherent possibility of bias. Secondly, there were more cystoscopies in the month preceding the biopsy in the CIP group, which is a post-PNB infection risk factor that could influence the incidence rate of urosepsis. However, this statistical difference was taken into account in the multivariate analysis. Finally, even if patients were told to seek medical attention at both our two institutions if specific symptoms occurred, it remains possible that patients consulted elsewhere (i.e. family physician, other hospitals) or did not seek medical care for minor complications. Our data set may underestimate the true frequency of the total post-PNB complications, particularly uncomplicated UTI. However, we have no reason to believe that rates of consultations to other centers would change during our study period.

\section{Conclusions}

In conclusion, our results show that the antibiotic prophylaxis combination of ciprofloxacin and fosfomycin tromethamine significantly lowered urosepsis rates following PNB. Benefits of this combination include single dose, low cost, and dodging the logistical burden of rectal swab screening and/or those associated with intravenous antibiotic prophylaxis administration. Further studies are needed to validate our findings and to evaluate the differences in the incidence of nonurosepsis complications. 


\section{References}

1. Zani EL, Clark OAC and Rodrigues Netto N: Antibiotic prophylaxis for transrectal prostate biopsy. Cochrane Database Syst Rev 2011: CD006576.

2. Liss MA, Ehdaie B, Loeb S, et al: An Update of the American Urological Association White Paper on the Prevention and Treatment of the More Common Complications Related to Prostate Biopsy. J. Urol. 2017; 198: 329-334.

3. Roberts MJ, Bennett HY, Harris PN, et al: Prostate Biopsy-related Infection: A Systematic Review of Risk Factors, Prevention Strategies, and Management Approaches. Urology 2017; 104: 11-21.

4. Carignan A, Roussy J-F, Lapointe V, et al: Increasing risk of infectious complications after transrectal ultrasound-guided prostate biopsies: time to reassess antimicrobial prophylaxis? Eur. Urol. 2012; 62: 453-459.

5. Nam RK, Saskin R, Lee Y, et al: Increasing hospital admission rates for urological complications after transrectal ultrasound guided prostate biopsy. J. Urol. 2013; 189: S1217; discussion S17-18.

6. Zaytoun OM, Vargo EH, Rajan R, et al: Emergence of fluoroquinolone-resistant Escherichia coli as cause of postprostate biopsy infection: implications for prophylaxis and treatment. Urology 2011; 77: 1035-1041.

7. Toner L, Bolton DM and Lawrentschuk N: Prevention of sepsis prior to prostate biopsy. Investigative and Clinical Urology 2016; 57: 94.

8. Zembower TR, Maxwell KM, Nadler RB, et al: Evaluation of targeted antimicrobial prophylaxis for transrectal ultrasound guided prostate biopsy: a prospective cohort trial. BMC Infect. Dis. 2017; 17: 401.

9. Adibi M, Hornberger B, Bhat D, et al: Reduction in Hospital Admission Rates Due to Post-Prostate Biopsy Infections After Augmenting Standard Antibiotic Prophylaxis. The Journal of Urology 2013; 189: 535-540.

10. Cai T, Gallelli L, Cocci A, et al: Antimicrobial prophylaxis for transrectal ultrasoundguided prostate biopsy: fosfomycin trometamol, an attractive alternative. World J Urol 2017; 35: 221-228.

11. Roberts MJ, Scott S, Harris PN, et al: Comparison of fosfomycin against fluoroquinolones for transrectal prostate biopsy prophylaxis: an individual patient-data meta-analysis. World J Urol 2018; 36: 323-330.

12. Kandil H, Cramp E and Vaghela T: Trends in Antibiotic Resistance in Urologic Practice. Eur Urol Focus 2016; 2: 363-373.

13. Loeb S, van den Heuvel S, Zhu X, et al: Infectious complications and hospital admissions after prostate biopsy in a European randomized trial. Eur. Urol. 2012; 61: 1110-1114.

14. Singer M, Deutschman CS, Seymour CW, et al: The Third International Consensus Definitions for Sepsis and Septic Shock (Sepsis-3). JAMA 2016; 315: 801-810.

15. Kapoor DA, Klimberg IW, Malek GH, et al: Single-dose oral ciprofloxacin versus placebo for prophylaxis during transrectal prostate biopsy. Urology 1998; 52: 552-558.

16. Bostwick DG and Meiers I: Prostate biopsy and optimization of cancer yield. Eur. Urol. 2006; 49: 415-417. 
17. Ohkoshi Y, Sato T, Suzuki Y, et al: Mechanism of Reduced Susceptibility to Fosfomycin in Escherichia coli Clinical Isolates. Biomed Res Int 2017; 2017: 5470241.

18. Karlowsky JA, Denisuik AJ, Lagacé-Wiens PRS, et al: In Vitro activity of fosfomycin against Escherichia coli isolated from patients with urinary tract infections in Canada as part of the CANWARD surveillance study. Antimicrob. Agents Chemother. 2014; 58: $1252-1256$.

19. Lista F, Redondo C, Meilán E, et al: Efficacy and safety of fosfomycin-trometamol in the prophylaxis for transrectal prostate biopsy. Prospective randomized comparison with ciprofloxacin. Actas Urol Esp 2014; 38: 391-396.

20. Carignan A, Sabbagh R, Masse V, et al: Effectiveness of fosfomycin tromethamine prophylaxis in preventing infection following transrectal ultrasound-guided prostate needle biopsy: Results from a large Canadian cohort. J Glob Antimicrob Resist 2018; 17: $112-116$.

21. Jiang P, Liss MA and Szabo RJ: Targeted Antimicrobial Prophylaxis Does Not Always Prevent Sepsis after Transrectal Prostate Biopsy. J. Urol. 2018; 200: 361-368.

22. Dai J, Leone A, Mermel L, et al: Rectal swab culture-directed antimicrobial prophylaxis for prostate biopsy and risk of postprocedure infection: a cohort study. Urology 2015; 85: 8-14.

23. Walker JT, Singla N and Roehrborn CG: Reducing Infectious Complications Following Transrectal Ultrasound-guided Prostate Biopsy: A Systematic Review. Rev Urol 2016; 18: 73-89.

24. Womble PR, Dixon MW, Linsell SM, et al: Infection related hospitalizations after prostate biopsy in a statewide quality improvement collaborative. J. Urol. 2014; 191 : 1787-1792.

25. Liss MA, Johnson JR, Porter SB, et al: Clinical and microbiological determinants of infection after transrectal prostate biopsy. Clin. Infect. Dis. 2015; 60: 979-987.

26. Bailey JK, Pinyon JL, Anantham S, et al: Commensal Escherichia coli of healthy humans: a reservoir for antibiotic-resistance determinants. J. Med. Microbiol. 2010; 59: 13311339.

27. Nielsen KL, Dynesen P, Larsen P, et al: Faecal Escherichia coli from patients with E. coli urinary tract infection and healthy controls who have never had a urinary tract infection. J. Med. Microbiol. 2014; 63: 582-589.

28. Xiang J, Yan H, Li J, et al: Transperineal versus transrectal prostate biopsy in the diagnosis of prostate cancer: a systematic review and meta-analysis. World J Surg Oncol 2019; 17: 31 .

29. McGrath S, Christidis D, Clarebrough E, et al: Transperineal prostate biopsy - tips for analgesia. BJU Int. 2017; 120: 164-167.

30. Meyer AR, Joice GA, Schwen ZR, et al: Initial Experience Performing In-office Ultrasound-guided Transperineal Prostate Biopsy Under Local Anesthesia Using the PrecisionPoint Transperineal Access System. Urology 2018; 115: 8-13. 
Figures and Tables

\begin{tabular}{|c|c|c|c|}
\hline Risk factors & $\begin{array}{l}\text { Ciprofloxacin } \\
(\%), n=1090\end{array}$ & $\begin{array}{c}\text { Ciprofloxacin and } \\
\text { fosfomycin (\%), } \\
n=1197\end{array}$ & $\mathbf{p}$ \\
\hline Age, yr, average \pm SD & $65.2 \pm 7.7$ & $65.0 \pm 7.5$ & 0.6 \\
\hline \multicolumn{4}{|l|}{ Infection risk factors } \\
\hline $\begin{array}{l}\text { Urinary tract infections in the last } 6 \\
\text { months }\end{array}$ & $4(0.4)$ & $12(1.0)$ & 0.08 \\
\hline $\begin{array}{l}\text { Extended-spectrum beta lactamase } \\
\text { stool sample in the last } 6 \text { months }\end{array}$ & - & $1(0.1)$ & - \\
\hline $\begin{array}{l}\text { C. difficile stool sample in the last } 6 \\
\text { months }\end{array}$ & & & - \\
\hline Hospitalized in the previous month & $19(1.7)$ & $18(1.5)$ & 0.7 \\
\hline \multicolumn{4}{|l|}{ Comorbidities } \\
\hline Diabetes & $132(12.1)$ & $142(11.7)$ & 0.9 \\
\hline Chronic obstructive pulmonary disease & $23(2.1)$ & $35(2.9)$ & 0.2 \\
\hline Heart valve & $8(0.7)$ & $4(0.3)$ & 0.2 \\
\hline Coronary artery disease & $86(7.9)$ & $79(6.6)$ & 0.2 \\
\hline \multicolumn{4}{|l|}{ Urological risk factors } \\
\hline Cystoscopy in the last month & $47(4.3)$ & $31(2.6)$ & 0.02 \\
\hline Urinary catheter in the last month & $6(0.6)$ & $5(0.4)$ & 0.6 \\
\hline Benign prostatic hyperplasia & $448(41.1)$ & $500(41.8)$ & 0.7 \\
\hline $\begin{array}{l}\text { Prostatic-specific antigen level, } \\
\mathrm{mg} / \mathrm{ml}(\mathrm{Q} 1 ; \mathrm{Q} 2 ; \mathrm{Q} 3)^{\mathrm{a}}\end{array}$ & $4.4 ; 5.7 ; 7.8$ & $4.7 ; 6.0 ; 8.2$ & 0.001 \\
\hline Repeat biopsy & $403(37.0)$ & $374(31.2)$ & 0.004 \\
\hline Prostate cancer histological finding & $626(57.4)$ & $773(64.6)$ & $<0.001$ \\
\hline
\end{tabular}

Data in parenthesis are percentages unless otherwise specified. ${ }^{a}$ Quartiles were used because prostate-specific antigen data did not follow a standard deviation. SD: standard deviation; Q1;Q2;Q3: quartile 1, 2, and 3 


\begin{tabular}{|l|c|c|c|}
\hline Table 2. Microbiological characteristics of urosepsis patient during hospitalization \\
\hline Group & Ciprofloxacin (\%) & $\begin{array}{c}\text { Ciprofloxacin and } \\
\text { fosfomycin (\%) }\end{array}$ & p \\
\hline Urosepsis & $12(1.1)$ & $2(0.2)$ & 0.02 \\
\hline Bacteremia & $7(0.6)$ & $1(0.1)$ & 0.07 \\
\hline Escherichia coli & 7 & 0 & \\
\hline $\begin{array}{l}\text { Fluoroquinolone- } \\
\text { resistant E. coli }\end{array}$ & 5 & 0 & \\
\hline ESBL E. coli & 2 & 0 & \\
\hline Bacterö̈des Fragilis & 0 & 1 & \\
\hline Bacteriuria & $11(1.0)$ & 1 & \\
\hline Escherichia coli & 11 & 0 & \\
\hline $\begin{array}{l}\text { Fluoroquinolone- } \\
\text { resistant E. coli }\end{array}$ & 7 & 0 & \\
\hline ESBL E. coli & 2 & $0.1)$ & \\
\hline
\end{tabular}

Data in parenthesis are percentages unless otherwise specified. ESBL: extended-spectrum betalactamase.

\begin{tabular}{|l|c|c|}
\hline \multicolumn{3}{|c|}{ Table 3. Non-infectious complications after prostate-biopsy } \\
\hline Group & $\begin{array}{c}\text { Ciprofloxacin } \\
(\%)\end{array}$ & $\begin{array}{c}\text { Ciprofloxacin } \\
\text { and fosfomycin } \\
(\%)\end{array}$ \\
\hline Grade 1 & $4(0.4)$ & $1(0.1)$ \\
\hline Macrohematuria & 3 & 1 \\
\hline Dysuria & 1 & - \\
\hline Hematospermia & - & - \\
\hline Grade 2 & $10(0.9)$ & $3(0.3)$ \\
\hline Acute urinary retention & 5 & 2 \\
\hline Rectal bleeding & 4 & 1 \\
\hline Prostatic hematoma & 1 & - \\
\hline
\end{tabular}

Data in parenthesis are percentages unless otherwise specified. 